\section{Current application of high flow oxygen nasal cannula in acute hypoxemic respiratory failure in the emergency department}

\author{
Giulia Bottani, Fabio Centurioni, Giacomo \\ Veronese, Fabrizio Vincenti, Elisa Forni, \\ Andrea Bellone
}

Department of Emergency Medicine, Niguarda Hospital, Milano, Italy

\begin{abstract}
High flow oxygen with nasal cannula (HFONC) is a relatively new mode of oxygen delivery. Advantages of HFONC versus conventional oxygen therapy (COT) encompass carbon dioxide washout, generation of a slight positive end-expiratory pressure and maintenance of humidified gas flow through airways. These features are mostly shared with non-invasive mechanical ventilation (NIMV), although with lack of a clearly comparable efficacy. In the last few years, HFONC has gained interest as a third alternative to COT and NIMV in the management of acute hypoxemic respiratory failure in the critically ill patient, both in intensive care units and emergency departments. The aim of this article is to review indications, effects and existing evidence on HFONC, COT and NIMV in the setting of acute hypoxemic respiratory failure.
\end{abstract}

\section{Introduction}

Humidified high-flow oxygen via nasal cannula (HFONC) is a relatively new method to improve fraction of inspired oxygen (FiO2) in hypoxemic patients. ${ }^{1,2}$ Initially used in neonatological setting, as an alternative to continuous positive airway pressure (CPAP) ${ }^{3}$ HFONC has been later used in the critically ill adult, in post-extubation respiratory assistance, in periprocedural assistance (e.g. during bronchoscopy) and in hypoxemic patients, as an alternative to conventional oxygen therapy (COT) or non-invasive mechanical ventilation (NIMV). ${ }^{4-6}$ Aim of this article is to review the physiological basis of HFONC, the actual evidence about its efficacy and the main indications and controversies. This review will analyze existing evidence about gas exchange, respiratory work, need for escalation to more invasive respiratory support (e.g. endotracheal intubation), mortality and patients-reported efficacy on dyspnea and device tolerance.

\section{Why humidified high-flow oxygen via nasal cannula?}

COT allows administration of oxygen mixed with room air delivered through simple nasal cannula or face-masks, therefore significantly increasing fraction of inspired oxygen (FiO2) and aiming to increase arterial oxygen tension $(\mathrm{PaO} 2)$. Traditional limits of COT are unpredictability for fixed inspired concentration of oxygen (due to the open circuit mixed up with room air), difficulty in maintaining humidity of the inspired gas, particularly in hypoxemic patients who need high $\mathrm{FiO} 2$ (e.g. > 0.5, 6 $\mathrm{L} / \mathrm{min}$ or more), and the substantial lack of any effect on breathing dynamic and work of breathing. HFONC therapy is carried out using an air/oxygen blender, active humidifier, a single heated tube, and high diameter nasal prongs (Figure 1). It is able thus to deliver adequately heated and humidified oxygen at flows reaching $60-70 \mathrm{~L} / \mathrm{min}$ and to maintain a fixed FiO2. ${ }^{1,2,7}$

\section{Indications and contraindications}

HFONC is generally used for respiratory support in critically ill patients in varying clinical situations, such as: i) Neonatological setting, as initial respiratory support or as weaning from NIMV or endotracheal intubation; ${ }^{3}$ ii) Post-extubation, including following surgery; ${ }^{4}$ iii) Acute hypoxemic respiratory failure; ${ }^{5}$ iv) Pre-intubation oxygenation; v) Avoiding intubation in immunocompromised patients.

HFONC has also been used in hypercapnic respiratory failure for patients unable to tolerate non-invasive ventilation because of claustrophobia or intolerance of tight interface contact. ${ }^{1,2}$ Currently there is not sufficient, well-established evidence or criteria for the clinical application of HFONC as well as criteria for starting and stopping it, and indications for treatment escalation; similarly, absolute contraindications are also lacking. However, it is reasonable to consider not suitable for HFONC those patients with respiratory muscles insufficiency requiring pressure support ventilation (PSV), those who need higher levels of PEEP (e.g. in the setting of acute pulmonary edema), or those who need both PSV and PEEP (e.g. in the setting of chronic obstructive pulmonary disease). For the above mentioned conditions, the use of NIMV is recommended with a strong level of evidence. $^{8}$

\section{Effects of humidified high-flow} oxygen via nasal cannula on alveolar oxygenation

As previously described, HFONC has been used initially in the neonatological setting to increase oxygenation, as an alterna-
Correspondence: Andrea Bellone, Department of Emergency Medicine, ASST Grande Ospedale Metropolitano Niguarda, Piazza Ospedale Maggiore 3, 20162 Milano, Italy. Tel.: +39.02.64442447 - Fax: +39.02.64442339. E-mail: andrea.bellone@ospedaleniguarda.it

Key words: hypoxemic respiratory failure; high flow oxygen nasal cannula; emergency medicine.

Contributions: the authors contributed equally.

Conflict of interests: the authors declare no potential conflict of interest.

Received for publication: 5 September 2017 Revision received: 19 December 2017. Accepted for publication: 11 January 2018.

This work is licensed under a Creative Commons Attribution 4.0 License (by-nc 4.0).

C) Copyright G. Bottani et al., 2017

Licensee PAGEPress, Italy

Emergency Care Journal 2017; 13:7036

doi:10.4081/ecj.2017.7036

tive to CPAP in preterm infants. ${ }^{3}$ The first studies involving the use of HFONC in adults analyzed its efficacy in periprocedural oxygenation, as well as in the post-extubation period in ICU patients or after cardio-thoracic surgery. ${ }^{4-6}$ Later, HFONC has been investigated as a potential alternative to conventional oxygen therapy and NIMV in patients with acute hypoxemic respiratory failure, in order to evaluate if the theoretical advantage of its mechanism of action was associated with a significant increase in alveolar oxygenation compared to COT and/or if it was, at least, comparable with the known efficacy of NIMV. There are varied mechanisms through which HFONC devices affect the respiratory system and alter gas exchange. First of all, HFONC provides for washout of nasopharyngeal dead space, which facilitates pulmonary gas exchange, leading to $\mathrm{PaO} 2$ improvement and, to a minor extent, also to carbon dioxide washout. ${ }^{1,2}$ Secondly, humidification of oxygen flow plays a role in preserving pulmonary conductance and compliance, avoiding bronchoconstriction reaction provoked by cool dry air flow and mediated by muscarinic receptors. Humidification also prevents the negative effects of breathing dry air, which is known to reduce nasal mucociliary clearance causing excessive water loss from the mucosa, acute damage and inflammation, potentially leading to atelectasis. ${ }^{9-12}$ Moreover, since in physiological condition inspiratory air needs to be warmed from room temperature to $37^{\circ} \mathrm{C}$ and humidified to $100 \%$ relative humidity 
by nasal mucosa, heat energy is required both to heat the air and to vaporize water into the air. Hence, heated and humidified high flow oxygen nasal cannulae produce a reduction in the metabolic cost of gas conditioning. ${ }^{13,14}$

Heat and humidification, along with the interface made by nasal prongs, are accountable for the great tolerance offered by the device. ${ }^{15,16}$ It is believed that high flow through the nasopharynx can be titrated to provide positive distending pressure in the airways, with a consequent slight increase of PEEP and end-expiratory lung volume. Although HFONC is an open system, high flow from the nasal cannula offers resistance against expiratory flow and increases airway pressure. Some studies demonstrate that at flows of $30-50 \mathrm{~L} / \mathrm{min}$ provided by HFONC system is able to provide a PEEP of 3-5 cm H2O, leading to clinical improvements. Parke et al. ${ }^{17}$ showed that there is substantial variation in pressure during inspiration and expiration demonstrating that the predominant benefits of positive pressure occur during expiration, particularly in patients who are at risk of, or have already established, atelectasis. It could be assumed that the mean expiratory pressure may be responsible for preventing atelectasis and that the peak and mean expiratory pressures may be responsible for reexpansion of collapsed areas. ${ }^{17}$ However, HFNOC are likely to deliver a clinically relevant PEEP level only if the patient's mouth is kept closed and the leak of flow around the nares is minimized. Corley et al. ${ }^{18}$ investigated the effect of HFONC on alveolar recruitment by means of measuring the relationship between airway pressure and endexpiratory lung volume using electrical impedance tomography. Compared with low-flow, HFONC significantly increased end-expiratory lung impedance and tidal impedance, suggesting an increase in endexpiratory lung volume and functional residual capacity (FRC). These improvements were translated into better oxygenation and decreased respiratory rate and dyspnea, with the greatest benefit in patients with a higher BMI. Consequently, it seems that at least part of the improvement in oxygenation observed in patients with acute respiratory failure is secondary to alveolar recruitment. Hence, due to its characteristics and mechanisms of action, HFONC has gained widespread use especially in the setting of acute hypoxemic respiratory failure. ${ }^{19}$ However, it is still debated whether the support provided by HFONC on work of breathing could be considered comparable to the one offered by NIMV. ${ }^{6}$ The strongest evidence is in favor of NIMV because of its proven ability to produce an ideal level of PEEP, especially in patients requiring high levels of PEEP. Therefore, numerous studies have been performed in order to evaluate the efficacy of HFONC, mostly compared with COT and/or NIMV.

\section{High flow oxygen with nasal cannu- la vs conventional oxygen therapy}

\section{Oxygenation and gas exchange}

Most studies investigating HFONC effect and comparison to other oxygenation techniques focus on $\mathrm{PaO} 2$ and its derivatives $\mathrm{PaO} 2 / \mathrm{FiO} 2(\mathrm{P} / \mathrm{F})$ and peripheral oxygen saturation $(\mathrm{SpO} 2){ }^{15}$ The vast majority of the studies, mostly prospective and observational, showed a sharp statistically significant benefit of HFONC versus COT in $\mathrm{PaO} 2$ after treatment. ${ }^{15,16,20-23}$ Oddly, the better designed and with higher populationenrolled study (FLORALI study, ${ }^{24}$ multicenter randomized and controlled) is the only one showing a slight but significant superiority of COT after 6 hours on gas exchanges. Current meta-analyses found in literature ${ }^{25,26}$ analyzed a mixed population (e.g. patients with AHRF, peri-procedural HFONC, post-surgery HFNC) and are therefore not completely reliable to determine whether HFONC is actually better or at least equivalent to $\mathrm{COT}$ in ameliorating oxygenation in patients with AHRF.

\section{Work of breathing}

The main analyzed parameter involving respiratory work is represented by respiratory rate (RR), mostly expressed as the absolute difference in breathes per minute between baseline and study (with the excep- tion of Bell et al. ${ }^{27}$ which accounted for a reduction of $\mathrm{RR}>20 \%$ from baseline). Among the studies commonly included in systematic reviews, seven ${ }^{5,15,20,21,23,24,28}$ show a statistically significant difference of RR between HFONC-group and COT-groups, in favor of HFONC, while no studies show a statistically significant difference in RR in favor of COT. ${ }^{29}$ The mechanical pattern of breathing was mainly expressed as thoracoabdominal synchrony and was analyzed in only three of these studies. Sztrymf et al. ${ }^{5}$ have studied thoraco-abdominal asynchrony and supraclavicular retraction, determining a progressive improvement in time (minutes to hours) after HFONC initiation. The second study by Itagaki et al. ${ }^{21}$ investigated work of breathing with both clinical and quantitative parameters (ratio of maximum compartmental amplitude to tidal volume and related phase angle), showing a significant improvement of breathing mechanics with HFONC vs COT. The third study ${ }^{23}$ investigated respiratory effort quantitatively (analyzing esophageal pressure-time product per minute) and showed improvements of respiratory effort vs Venturi mask, but not vs CPAP (set at 5 $\mathrm{cmH} 2 \mathrm{O}$ ). In conclusion, limited available data seem to support efficacy of HFONC in ameliorating work of breathing vs COT.

\section{High flow oxygen with nasal cannu- la vs non-invasive mechanical ventilation}

\section{Oxygenation and gas exchange}

NIMV is considered superior to HFONC in hypercapnic respiratory failure,

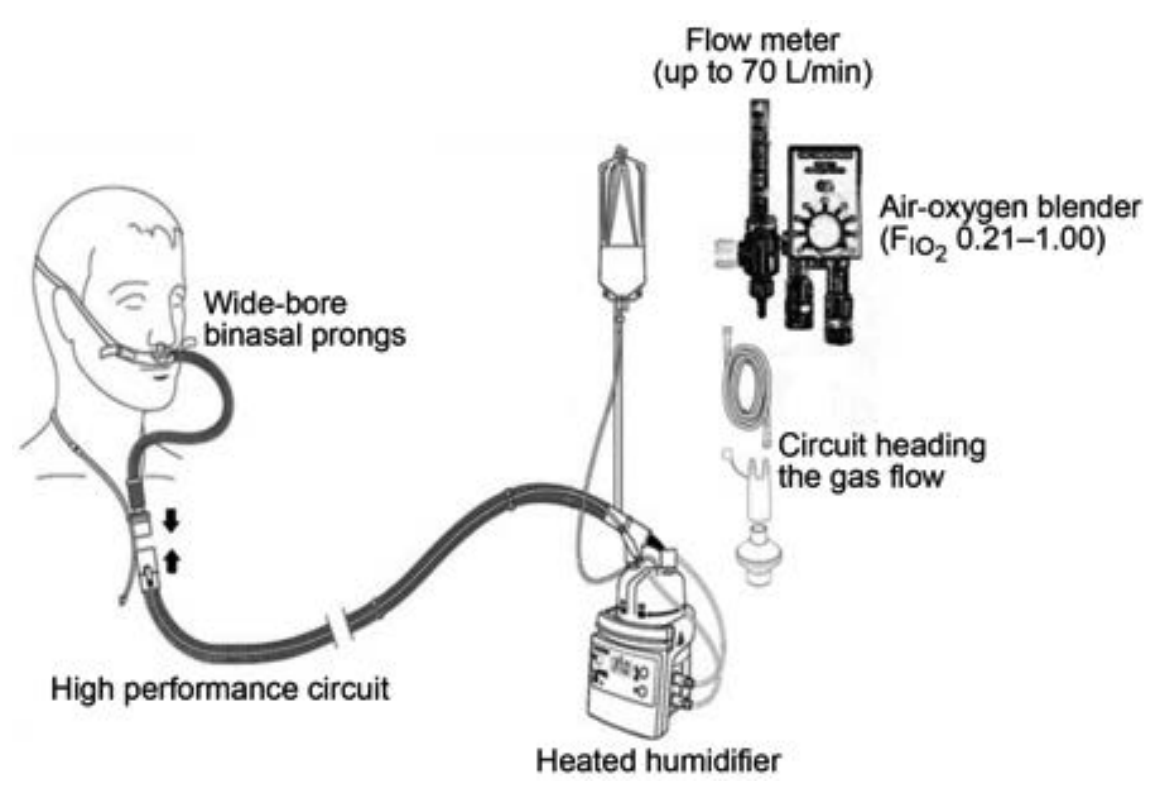

Figure 1. Humidified high-flow oxygen via nasal cannulae device. 
due to its greater ability to eliminate carbon dioxide and to support work of breathing. ${ }^{30}$ Indeed, one of the most common exclusion criteria for studies involving HFONC was a $\mathrm{PaCO} 2$ higher than $45 \mathrm{mmHg}{ }^{6}$ The majority of literature compared HFONC and NIMV in selected clinical situations (mostly post or peri-extubation and in the neonatological setting). ${ }^{25,26}$ Only a few articles investigated its value in the general management of AHRF (e.g. AHRF in an adult in the ED or inpatient). To our knowledge, only four studies investigated this feature. Amongst these, the FLORALI study ${ }^{24}$ again is the most important because of its design and the large enrolled population, with the other three ${ }^{22,23,31}$ being limited by the small number of subjects involved (14, 28 and 12 respectively). In the FLORALI study, $\mathrm{PaO} 2$ and $\mathrm{P} / \mathrm{F}$ were compared between HFONC and NIMV at 1 and 6 hours from randomization, showing significantly higher $\mathrm{PaO} 2$ and $\mathrm{P} / \mathrm{F}$ for NIMV-group in both. Schwabbauer et al. ${ }^{22}$ and Vargas et al. ${ }^{23} \mathrm{did}$ not reach statistical significance, while Frat et al. ${ }^{24}$ concluded for an increase in $\mathrm{P} / \mathrm{F}$ only in the NIMV-group. It seems reasonable to assume that NIMV is actually superior to HFONC in increasing $\mathrm{PaO} 2$ and $\mathrm{P} / \mathrm{F}$ in adults with AHRF.

\section{Work of breathing}

Three studies $22-24$ compared RR between HFONC and NIMV: among these, the only significant difference found was an initial and temporary improvement of RR in HFONC-group at 1 hour from beginning of administration, which was lost at the sixhours control, in the FLORALI study.

\section{Dyspnea and device tolerance}

Assessment of dyspnea has been carried out in most RCTs using a combination of visual analog scale, visual numerical scale, five point Likert Scale, and Borg scale. Although rarely reaching significance, the majority of the studies showed improved dyspnea, comfort and tolerability scores in favor of HFONC compared with NIMV and COT. ${ }^{5,15,20,32-35}$ HFONC has been reported to lead to rapid alleviation of respiratory distress in more severe patients. ${ }^{28}$ Its remarkable tolerance during acute hypoxemic respiratory failure allows a much longer duration of use, being attributable partly to the heat and humidity supplied by the device and partly to the comfort given by its interface. ${ }^{15,16}$

\section{Endotracheal intubation and mortality}

The two main questions that still remain without a definite answer are whether or not HFONC reduces mortality and need for intubation in patients with hypoxemic acute respiratory failure. Overall, no significant difference in mortality or intubation rate was detected in adult patients with acute respiratory failure treated with $\mathrm{HFONC}$, when compared with usual care defined as COT or NIMV, so that HFONC can be considered at least non-inferior to NIMV with regard to these outcomes. ${ }^{6,26,36-41}$ As for the intubation rate, there is only one RCT showing a significant difference in favor of HFONC: ${ }^{24}$ in the post hoc analyses, the authors showed HFONC to be significantly superior in reducing endotracheal intubation when compared with COT alone, and also superior when compared to both COT and NIMV in the subgroup with severe hypoxemic respiratory failure $(\mathrm{P} / \mathrm{F}$ ratio $<200 \mathrm{~mm} \mathrm{Hg}$ ). The study also showed a significant reduction in duration of mechanical ventilation and 90-day mortality in the HFONC group as secondary outcomes. The other studies did not find any significant difference with respect either to mortality and endotracheal intubation rate. Some studies have suggested that time to intubation may be longer in patients treated with HFONC compared with COT. ${ }^{39}$ In patients treated with NIMV, the time to intubation resulted to be similar in comparison with HFONC patients. ${ }^{24,36}$ This could reflect the existing major controversy regarding the use of NIMV in AHRF stating that prosecution of NIMV, aimed to avoid intubation, paradoxically results in delayed intubation which in turn leads to increased mortality.

\section{Discussion}

Available evidence is insufficient to determine whether HFONC is actually superior to COT in the treatment of AHRF. ${ }^{6,24-26}$ This is due to multiple factors encompassing strong differences in design, primary outcomes and demography of the population enrolled in studies, as stated in almost all available reviews. As an example, it is useful to consider two studies of the same author. ${ }^{24,31}$ Frat's first observational sequential pilot study enrolled 28 patients, mostly with a diagnosis of ARDS, and showed a substantial benefit of HFONC vs $\mathrm{COT}$ in $\mathrm{PaO} 2$ after the first hours of treatment. The FLORALI study, ${ }^{24}$ as already described, was designed as a RCT enrolling 310 patients mostly with a diagnosis of pneumonia and showed the opposite results. It is clear that a different pathophysiology may, at least partially, explain the differences in these findings. Of note, it is worth to mention that the primary outcome was not oxygenation but rate of intubation. More recent studies are trying to avoid this bias by recruiting patients based on the operative diagnosis, rather than recruiting patients with AHRF from various etiologies: Mackdee et al. ${ }^{28}$ for instance found that in AHRF due to congestive heart failure in the ED, HFONC may decrease the severity of dyspnea during the first hour of treatment, when compared to COT.

\section{Conclusions}

In conclusion, due to its safety, efficacy and tolerability, we suggest that HFONC is a very promising technique, currently representing a valuable alternative to NIMV in selected patients with AHRF. However, HFONC non-inferiority to NIMV has not yet been clearly demonstrated and may be thus considered a half way treatment between COT and NIMV. Key elements for its right application are a correct etiological diagnosis of respiratory failure and a careful selection of patients. Further studies on homogeneous populations are warranted in order to provide additional evidence for the application of this technique.

\section{References}

1. Nishimura M. High-flow nasal cannula oxygen therapy in adults: physiological benefits, indication, clinical benefits, and adverse effects. Respiratory Care 2016;61:529-41.

2. Dysart K, Miller TL, Wolfson MR, Shaffer TH. Research in high flow therapy: mechanisms of action. Respir Med 2009;103:1400-5.

3. Spence KL, Murphy D, Kilian C, et al. High-flow nasal cannula as a device to provide continuous positive airway pressure in infants. J Perinatol 2007;27:772-5.

4. Futier E, Jaber S. High-flow nasal cannula following extubation: is more oxygen flow useful after surgery? Intensive Care Med 2015; 41:1310-3.

5. Sztrymf B, Messika J, Bertrand F, et al. Beneficial effects of humidified high flow nasal oxygen in critical care patients: a prospective pilot study. Intensive Care Med 2011;37:1780-6.

6. Lee CC, Mankodi D, Shaharyar S, et al. High flow nasal cannula versus conventional oxygen therapy and non-invasive ventilation in adults with acute hypoxemic respiratory failure: a systematic review. Respir Med 2016;121:100-8.

7. Gotera C, Díaz Lobato S, Pinto T, et al. Clinical evidence on high flow oxygen therapy and active humidification in adults. Rev Port Pneumol 2013;19:217- 
27.

8. Rochwerg B, Brochard L, Elliott MW, et al. Official ERS/ATS clinical practice guidelines: noninvasive ventilation for acute respiratory failure. Eur Respir J 2017;31:50.

9. Salah B, Dinh Xuan AT, Fouilladieu JL, et al. Nasal mucociliary transport in healthy subjects is slower when breathing dry air. Eur Respir J 1988; 1:852-5.

10. Barbet JP, Chauveau M, Labbé S, Lockhart A. Breathing dry air causes acute epithelial damage and inflammation of the guinea pig trachea. J Appl Physiol 1988;64:1851-7.

11. Kilgour E, Rankin N, Ryan S, et al. Mucociliary function deteriorates in the clinical range of inspired air temperature and humidity. Intensive Care Med 2004;30:1491-4

12. Spoletini G, Alotaibi M, Blasi F, Hill NS. Heated humidified high-flow nasal oxygen in adults. Chest 2015; 148:25361.

13. Fontanari $P$, Burnet $H$, ZattaraHartmann MC, Jammes Y. Changes in airway resistance induced by nasal inhalation of cold dry, dry, or moist air in normal individuals. J Appl Physiol 1996;81:1739-43.

14. Fontanari P, Zattara-Hartmann M, Burnet H, Jammes Y. Nasal eupnoeic inhalation of cold, dry air increases airway resistance in asthmatic patients. Eur Respir J 1997;10:2250-4.

15. Roca O, Riera J, Torres F, Masclans JR. High-flow oxygen therapy in acute respiratory failure. Respir Care 2010;55:408-13.

16. Rittayamai N, Tscheikuna J. Ruijwit P. High-flow nasal cannula versus conventional oxygen therapy after endotracheal extubation: a randomized crossover physiologic study. Respir Care 2014;59:485-90.

17. Parke RL, Mc Guinness SP. Pressures delivered by nasal high flow oxygen during all phases of the respiratory cycle. Respir Care 2013;58:1621-4.

18. Corley A, Caruana LR, Barnett AG, et al. Oxygen delivery through high-flow nasal cannulae increase end-expiratory lung volume and reduce respiratory rate in post-cardiac surgical patients. $\mathrm{Br} \mathrm{J}$ Anaesth 2011;107:998-1004.

19. Ricard JD. High flow nasal oxygen in acute respiratory failure. Minerva Anestesiol 2012;78:836-41.

20. Sztrymf B, Messika J, Mayot T, et al. Impact of high-flow nasal cannula oxygen therapy on intensive care unit patients with acute respiratory failure: a prospective observational study. J Crit Care 2012;27:324.e9-13.
21. Itagaki T, Okuda N, Tsunano Y, et al. Effect of high flow nasal cannula on thoraco-abdominal synchrony in adult critically ill patients. Respir Care 2014;59:70-4.

22. Schwabbauer N, Berg B, Blumenstock $\mathrm{G}$, et al. Nasal high-flow oxygen therapy in patients with hypoxic respiratory failure: effect on functional and subjective respiratory parameters compared to conventional oxygen therapy and noninvasive ventilation (NIV). BMC Anesthesiol 2014;7:66.

23. Vargas F, Saint-Leger M, Boyer A, et al. Physiologic effects of high-flow nasal cannula oxygen in critical care subjects. Respir Care 2015;60:1369-76.

24. Frat JP, Thille AW, Mercat A, et al. High-flow oxygen through nasal cannula in acute hypoxemic respiratory failure. N Engl J Med 2015;372: 2185-96.

25. Nedel WL, Deutschendorf C, Moraes Rodrigues Filho E. High-flow nasal cannula in critically ill subjects with or at risk for respiratory failure: a systematic review and meta-analysis. Respir Care 2017;62:123-32.

26. Ou X, Hua Y, Liu J, et al. Effect of highflow nasal cannula oxygen therapy in adults with acute hypoxemic respiratory failure: a meta-analysis of randomized controlled trials. CMAJ 2017;189:E260-7.

27. Bell N, Hutchinson CL, Green TC, et al. Randomised control trial of humidified high flow nasal cannulae versus standard oxygen in the emergency department. Emerg Med Australas 2015;27:537-41.

28. Makdee O, Monsomboon A, Surabenjawong U, et al. High-flow nasal cannula versus conventional oxygen therapy in emergency department patients with cardiogenic pulmonary edema: a randomized controlled trial. Ann Emerg Med 2017;70:465-72.

29. Parke RL, McGuiness SP, Eccleston ML. A preliminary randomized controlled trial to assess effectiveness of nasal high-flow oxygen in intensive care patients. Respir Care 2011;56: 265 70.

30. Corley A, Rickard C, Aitken LM, et al. High flow nasal cannulae for respiratory support in adult intensive care patients. Cochrane Database Syst Rev 2017;30:5.

31. Frat JP, Brugiere B, Ragot S. Sequential application of oxygen therapy via highflow nasal cannula and noninvasive ventilation in acute respiratory failure: an observational pilot study. Respir Care 2015;60:170-8.

32. Rittayamai N, Tsecheikuna J,
Praphruetkit N, Kijpinyochai S. Use of high-flow nasal cannula for acute dyspnea and hypoxemia in the emergency department. Respir Care 2015;6:137782.

33. Cuquemelle E, Pham T, Papon JF, et al. Heated and humidified highflow oxygen therapy reduces discomfort during hypoxemic respiratory failure. Respir Care 2012;57:1571-7.

34. Maggiore SM, Idone FA, Vaschetto R, et al. Nasal high-flow versus Venturi mask oxygen therapy after extubation. Effects on oxygenation, comfort, and clinical outcome. Am J Respir Crit Care Med 2014;190:282-8.

35. Hui D, Morgado M, Chisholm G, et al. High-flow oxygen and bilevel positive airway pressure for persistent dyspnea in patients with advanced cancer: a phase II randomized trial. J Pain Symptom Manage 2013;46:463-73.

36. Stéphan F, Barrucand B, Petit P, et al. High-flow nasal oxygen vs noninvasive positive airway pressure in hypoxemic patients after cardiothoracic surgery: a randomized clinical trial. JAMA 2015; 313:2331-9.

37. Monro-Somerville T, Sim M, Ruddy J, et al. The effect of high-flow nasal cannula oxygen therapy on mortality and intubation rate in acute respiratory failure: a systematic review and metaanalysis. Crit Care Med 2017; 45:e44956.

38. Tiruvoipati R, Lewis D, Haji K, Botha J. High-flow nasal oxygen vs highflow face mask: a randomized crossover trial in extubated patients. J Crit Care 2010;25:463-8.

39. Parke R, McGuinness S, Dixon R, Jull A. Open-label, phase II study of routine high-flow nasal oxygen therapy in cardiac surgical patients. $\mathrm{Br} \mathrm{J}$ Anaesth 2013;111:925-31.

40. Lemiale V, Mokart D, Mayaux J, et al. The effects of a 2-h trial of high-flow oxygen by nasal cannula versus Venturi mask in immunocompromised patients with hypoxemic acute respiratory failure: a multicenter randomized trial. Crit Care 2015;19:380.

41. Corley A, Bull T, Spooner AJ, et al. Direct extubation onto high-flow nasal cannulae post-cardiac surgery versus standard treatment in patients with a $\mathrm{BMI} \geq 30$ : a randomised controlled trial. Intensive Care Med 2015;41: 887-94. 